\title{
Antenna radiation enhanced by a negative permittivity material: plasma
}

\author{
Zhen Zhang, Chunsheng Wang ${ }^{\mathrm{a}}$ and Qiuyue Nie \\ Harbin Institute of Technology, School of Electrical Engineering \& Automation, Harbin, Heilongjiang, China
}

\begin{abstract}
As a kind of negative dielectric constant material, finite plasma has some effect on antenna radiation. Results show the plasma layer with negative permittivity is inductive, which can compensate the capacitivity of the vacuum and enhance the radiation with proper para-meters. Furthermore, the shape of plasma layer is changed to verify the effect of plasma boundary on antenna radiation. The study shows the effect of thin plasma layer on electromagnetic field and provides a type of plasma antenna.
\end{abstract}

Keywords: plasma layer, negative permittivity, radiation enhancement.

\section{Introduction}

It is well known that the antenna radiation will be cut off if an antenna is immersed in an infinite plasma with the plasma frequency exceeding the antenna frequency. However, when a finite plasma layer is considered, the effect of plasma layer on antenna radiation is complicated. For example, the plasma sheath around the re-entry vehicle generated by high speed flow can cause intensity attenuation, even interruption, of antenna radiation (called the blackout phenomenon) [1-2]. The blackout phenomenon has been an urgent problem to be solved in the communication of spaceplane. But, another phenomenon, the intensity of antenna radiation can be enhanced if the parameters of plasma layer are chosen properly, has been proved by numerical and experimental studies. This phenomenon shows a way to overcome the blackout problem [3-4]. So the effect of plasma layer on antenna radiation is determined by parameters of plasma layer.

The enhancement of thin plasma layer on radiation intensity of antenna was reported first by Messiaen and Vandenplas. Experimental results show the radiation of antenna can be enhanced if the antenna frequency is much lower than the plasma frequency and the antenna dimension are properly chosen [3]. Furthermore, the phenomenon was studied in a lossy hot plasma and some studies also considered the effect of a plasma sheath existing between the antenna surface and the plasma layer [4-5]. Ref.6 shown the influence of antenna frequency, plasma layer width and collision frequency on radiation intensity [6]. Also, the effect of plasma layer with different shape is considered [7].

The influence of single parameter on antenna radiation has been given. But without a unified scheme, the results cannot be combined and the exact conditions for the enhancement or attenuation of antenna radiation have not been given. In this letter, through analyzing on the energy propagation in the near-field

${ }^{a}$ Corresponding author: wangcs@hit.edu.cn 
of antenna radiation, the effect mechanism of plasma layer on antenna radiation is discovered. Furthermore, the effect of plasma shape on antenna radiation is discussed.

\section{Dielectric characteristics of plasma}

For a uniform dielectric, the time-harmonic electromagnetic field of an infinitesimal dipole in spherical coordinates can be expressed as [8],

$$
\begin{gathered}
\vec{E}=\eta \frac{I_{0} l \cos (\theta)}{2 \pi r^{2}}\left[1+\frac{1}{j k r}\right] e^{-j k r} \vec{e}_{r}+j \eta \frac{k I_{0} l \sin (\theta)}{4 \pi r}\left[1+\frac{1}{j k r}-\frac{1}{(k r)^{2}}\right] e^{-j k r} \vec{e}_{\theta} \\
\vec{H}=j \frac{k I_{0} l \sin (\theta)}{4 \pi r}\left[1+\frac{1}{j k r}\right] e^{-j k r} \vec{e}_{\phi}
\end{gathered}
$$

Where, $l$ is the distance of the infinitesimal dipoles and $I_{0}$ is the amplitude of excited current. $\eta=\sqrt{\mu / \varepsilon}$ is the wave impedance, $k=\omega \sqrt{\mu \varepsilon}$ is the wave number. $\varepsilon, \mu$ are the permittivity and permeability of the dielectric, respectively. The permittivity of uniform lossless plasma is [9],

$$
\varepsilon_{p}=\varepsilon_{0} \varepsilon_{r}=\varepsilon_{0}\left(1-\frac{\omega_{p}^{2}}{\omega^{2}}\right)
$$

Where, $\omega$ is the frequency of the antenna radiation, $\omega_{p}=\sqrt{n_{0} e^{2} /\left(\varepsilon_{0} m_{e}\right)}$ is the plasma frequency. When the plasma frequency is larger than antenna frequency, the permittivity of plasma is negative.

As the antenna is covered by plasma layer, we consider the energy propagation in the near-field of antenna radiation. The average power density can be expressed as

$$
S_{a v}=\frac{1}{2} \operatorname{Re}\left(\vec{E}_{n} \times \vec{H}_{n}^{*}\right)=\frac{1}{2}\left|E_{n}\right| \cdot\left|H_{n}\right| \cos \varphi_{E-H}
$$

Where, the electromagnetic field in the near-field ( $k r \ll 1$ ) of antenna radiation is quasi-stationary, as expressed by

$$
\begin{gathered}
\vec{E}_{n}=-j \frac{I_{0} l \cos (\theta)}{2 \pi \omega \varepsilon r^{3}} \vec{e}_{r}-j \frac{I_{0} l \sin (\theta)}{4 \pi \omega \varepsilon r^{3}} \vec{e}_{\theta} \\
\vec{H}_{n}=\frac{I_{0} l \sin (\theta)}{4 \pi r^{2}} \vec{e}_{\phi}
\end{gathered}
$$

For the dielectric of vacuum and plasma with negative permittivity, the amplitude of electromagnetic field is similar, which drops drastically with the increase of radial distance. But the phase-difference between electric and magnetic field is different. The vacuum is capacitive in the near-field region and the phase-difference is $-90^{\circ}$. With negative permittivity, the plasma layer is inductive and the phase-difference is $90^{\circ}$. Because the cosine of phase-difference between electric and magnetic field is zero, both the vacuum and plasma with negative permittivity, the average power density is zero. So, for 
single dielectric, the electrostatic energy was stored in the near-field of antenna radiation and the radiation intensity drop drastically.

\section{Numerical results and analyses}

\subsection{Model and numerical results}

The two-domain model in Ref. 5 is adopted to study the energy propagation of antenna radiation in two-layer dielectrics of plasma and vacuum (Fig.1). The model includes: a spherical antenna with radius of $r_{1}=0.6 \mathrm{~cm}$, a spherical plasma layer surrounde the antenna (the outer surface is at $r_{2}$ ), the infinite space surrounding the plasma layer is modeled by an air layer (the outer surface is at $r_{3}=100 \mathrm{~cm}$ ) and a perfect match layer $\left(d_{2}=5 \mathrm{~cm}\right)$. The spherical antenna is assumed to be a perfect electrical conductor and driven by a voltage of $V_{0}=10 \mathrm{~V}$, across a narrow gap $\left(d_{1}=0.03 \mathrm{~cm}\right)$ between the two poles of the antenna. The frequency of the antenna radiation is $\omega=0.6 \pi \mathrm{GHz}$.

With different plasma layer width, the influences of plasma density on radiation density are studied. At the observed point $\mathrm{P}(r=90 \mathrm{~cm})$, the antenna radiated power normalized by its free space radiated power is shown in Fig. 2 (the horizontal coordinate is relative plasma frequency $\omega_{p} / \omega$ ). It can be observed that the radiated power of antenna decrease firstly.

After the minimum at $\omega_{p}=\omega$, the radiated power increase and then decrease. When the width of plasma layer is larger than $22 \mathrm{~cm}$, there is a plasma frequency zone, where the relative radiated power is greater than zero. It shows the radiation enhancement phenomenon.

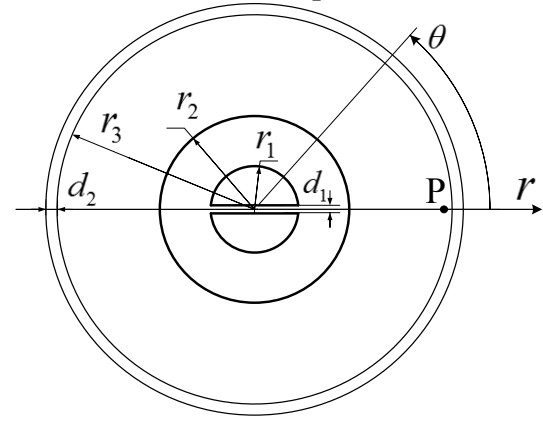

Figure 1. Geometry of this plasma-antenna system.

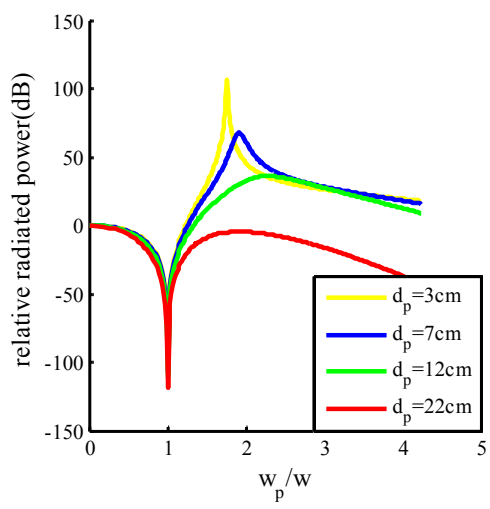

Figure 2. Effect of plasma frequency on the radiated power for different widths on the plasma width. 


\subsection{The Effect of plasma shape on radiation with plasma area unchanged}

The plasma layer acts as an antenna, which increases the radiant surface. To verify the effect of plasma boundary on electric field, the shape of plasma layer is changed to ellipse with the plasma area unchanged. A parameter $\alpha$ is introduced. The $\mathrm{z}$-axis is expressed as $r_{2} \times \alpha$ and the value of r-axis is $r_{2} / \alpha$. To study the effect mechanism of plasma shape on antenna radiation, the width of plasma layer $r_{2}$ is taken as $7 \mathrm{~cm}$. With different $\alpha$, the change of relative radiation intensity with relative plasma frequency is shown in FIG.3.

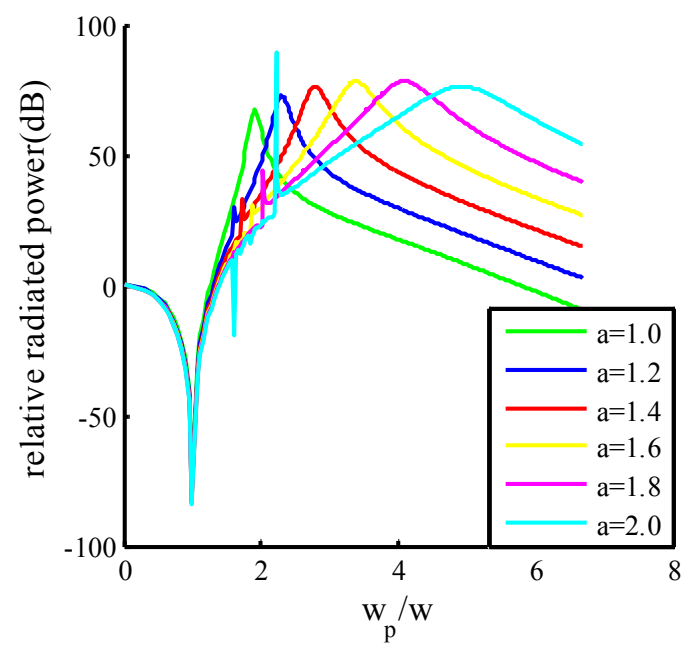

Figure 3. Changes of the relative radiated power with relative plasma frequency for different $\alpha$.

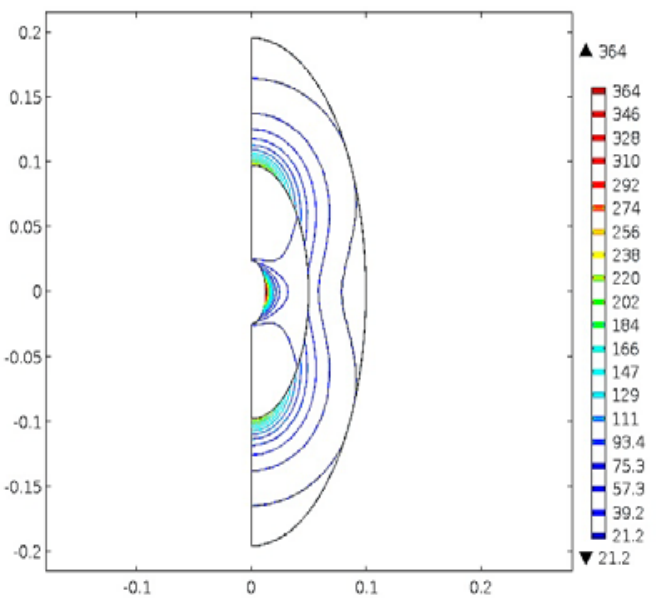

Figure 4. The contour of electric field intensity of $\alpha=1.4$ near the interface of plasma layer and air at the condition of maximum radiation intensity.

With the extension of the long axis of the ellipse, the radiation intensity and the plasma frequency associated with the maximum radiation intensity increase. The contour of electric field intensity of electric field intensity of $\alpha=1.4$ near the interface of plasma layer and air is shown in FIG.4. When the radiation intensity achieves maximum, an ellipse region of equal electric field is formed in plasma layer. Like cylindrical antenna, the electric field appears high at the ends of the long axis of the ellipse. 


\subsection{The effect of plasma shape on radiation with R-axis or Z-axis unchanged}

Furthermore, we consider the effect of plasma shape on radiation with r-axis or z-axis unchanged. The width of plasma layer is $r_{2}=7 \mathrm{~cm}$. There is a parameter $\alpha$. When r-axis is constant with the value of $r_{2}$, the $\mathrm{z}$-axis is expressed as $r_{2} \times \alpha$. With different $\alpha$, the change of relative radiation intensity with relative plasma frequency is shown in FIG.5.In the same way, the value of $r$-axis is $r_{2} \times \alpha$. The results are shown in FIG.6.

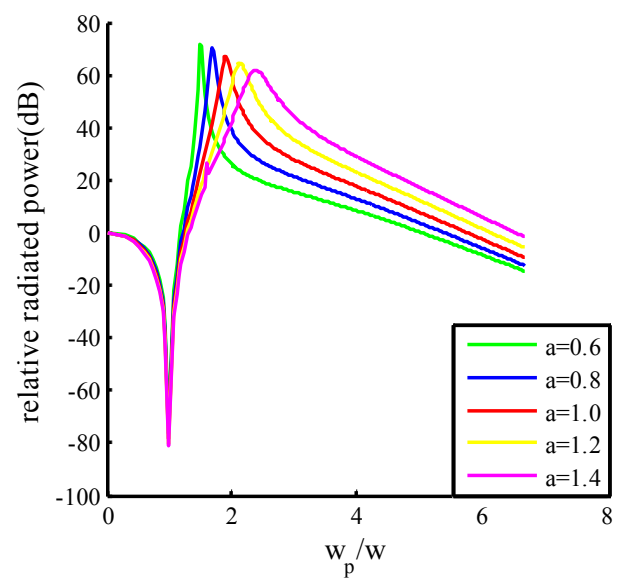

Figure 5. Changes of the relative radiated power with relative plasma frequency for different $\alpha$ with r-axis unchanged.

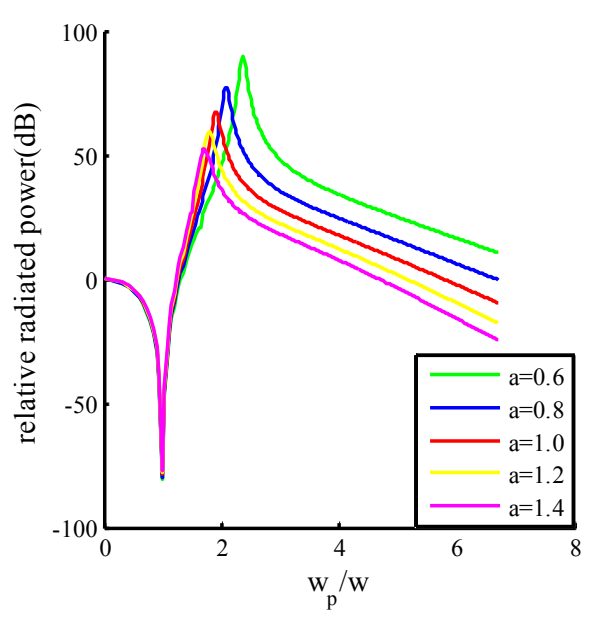

Figure 6. Changes of the relative radiated power with relative plasma frequency for different $\alpha$ with z-axis unchanged.

With the increase of the z-axis, the radiation intensity decrease, but it changes slowly, there is a wider range of radiation enhancement. while the plasma frequency associated with the maximum radiation intensity increase when $r$-axis is unchanged.

When z-axis is constant, there is a different result. With the extension of the r-axis, both the radiation intensity and the plasma frequency associated with the maximum radiation intensity decrease. 


\section{Conclusions}

The effect of plasma layer on the propagation of electromagnetic field is a common problem relating to many critical problem. For example, blackout problem, plasma antenna, plasma stealth and so on. This study discovers the characteristics of plasma as a negative permittivity materials and provides an efficient method to investigate associated problems.

The radiation power can be enhanced when the width of plasma layer is less than that of the near-field. With the increase of plasma density, the region of electrostatic field extends and the radiation intensity decreases. Furthermore, the radiation characteristics can be modified by the shape of plasma layer.

\section{Acknowledgments}

This work is funded by the National Natural Science Foundation of China (Grant No. 51207033).

\section{References}

1. M. Kim, M. Keidar, and I. D. Boyd, Analysis of an electromagnetic mitigation scheme for reentry telemetry through plasma,Journal of Spacecraft and Rockets, 45(6), 1223-1229 (2008).

2. R.A.Hartunian, G.E. Stewart, T.J. Curtiss, et al., Implications and mitigation of radio frequency blackout during reentry of reusable launch vehicles. AIAA Paper, 2007. 6633: p. 2007.

3. Chen, K. M. and C. C. Lin, Enhanced radiation from a plasma embedded antenna, Proc. IEEE, Vol. 56, 1595-1597, 1968.

4. C. C. Lin and K. M. Chen, Radiation from a spherical antenna covered by a layer of lossy hot plasma: Theory and experiment, Proc. Inst. Electr. Eng. 118(1), 36-42 (1971).

5. A. M. Messiaen and P. E. Vandenplas, Theory and experiments of the enhanced radiation from a plasma-coated antenna, Electron. Lett. 3, 26 (1967).

6. C. Lin and K. M. Chen, Improved radiation from a spherical antenna by overdense plasma coating, IEEE Trans. Antennas and Propag. 17(5), 675-678 (1969).

7. T. I. Bichutskaya and G. I. Makarov, Radiation from an electric dipole surrounded by a plasma cylinder with a cavity, Radiophys. Quantum Electron. 45(7), 509-519 (2002).

8. Balanis, C.A., Antenna theory: analysis and design. 2012: John Wiley \& Sons.

9. W. Rotman, Plasma simulation by artificial dielectrics and parallel-plate media, IRE Trans. Antennas Propag. 10(1), 82-95 (1962). 